\title{
Not all unexplained hypoxia is pulmonary embolism
}

\author{
Mui Teng $\underline{\text { Chua }}^{1,2}$, MBBS, MCEM, Tiong Beng $\underline{\text { Sim }}^{1,2}$, MBBS, MRCS, Irwani $\underline{\operatorname{Ibrahim}}^{1,2}$, MBBS, FRCS
}

\begin{abstract}
Acute myocardial infarction is one of the conditions frequently managed in the emergency department. There are many complications associated with right ventricular infarction, and the incidence of right ventricular infarction associated with inferior myocardial infarction is as high as $51 \%$ based on electrocardiographic findings. We herein report the case of a 45-year-old Chinese man with inferior myocardial infarction complicated by right ventricular failure. He had hypoxaemia refractory to supplemental oxygen due to an acute right-to-left shunting through a patent foramen ovale (PFO). He underwent coronary angioplasty and closure of the PFO. It is crucial for the attending physician to consider the presence of a right-to-left shunt when there is persistent uncorrectable hypoxaemia despite maximal oxygen supplementation in the setting of right ventricular infarction, as there are clinical implications and certain clinical managing principles that should be applied.
\end{abstract}

Keywords: acute myocardial infarction, acute right-to-left shunt, patent foramen ovale, persistent hypoxia, refractory hypoxia

\section{INTRODUCTION}

Acute myocardial infarction is a condition frequently managed in the emergency department, accounting up to $14.7 \%$ of visits for chest pain. ${ }^{(1)}$ The incidence of right ventricular (RV) infarction associated with inferior myocardial infarction is as high as $51 \%$ based on electrocardiographic findings ${ }^{(2)}$ and it can occur in isolation in less than $3 \%$ of infarcts. ${ }^{(3)} \mathrm{RV}$ infarction has many complications, ranging from arrhythmia to hypotension, and there have been described cases of persistent hypoxaemia from the development of an acute right-to-left shunting through a previously dormant patent foramen ovale (PFO). Herein, we report the case of a patient with inferior myocardial infarction complicated by RV failure and had refractory hypoxaemia due to an acute right-to-left shunting through a PFO.

\section{CASE REPORT}

A 45-year-old Chinese man presented to our emergency department (ED) with a two-day history of exertional shortness of breath, left-sided chest pain and abdominal bloating. He was a smoker and had a medical history of hypertension, hyperlipidaemia, and inferior and RV ST-elevation myocardial infarction (STEMI) ten years ago status post-percutaneous coronary intervention $(\mathrm{PCl})$ to the distal right coronary artery (RCA). Coronary angiography done three years after $\mathrm{PCI}$ showed a patent distal RCA stent. He subsequently defaulted his medications.

On arrival to our ED, his vital signs were as follows: blood pressure (BP) 88/59 $\mathrm{mmHg}$; respiratory rate 22 breaths per min; and oxygen saturation by pulse oximetry $90 \%$ on nonrebreather mask. There were decreased breath sounds bibasally with occasional basal crepitations. The jugular venous pressure was not elevated, and the rest of the physical examination was unremarkable.

Electrocardiography (ECG) showed evolved inferior STEMI (Figs. 1 \& 2), which was different from his previous ECG (Fig. 3).
Arterial blood gas revealed type I respiratory failure with partial oxygen pressure $\left(\mathrm{PaO}_{2}\right)$ of $50 \mathrm{mmHg}$ on room air. Laboratory findings showed elevated cardiac enzymes: creatine kinase 1029 (normal range [NR] 30-350) U/L, creatine kinase-MB 28.4 $(\mathrm{NR}$ 1.0-6.0) $\mu \mathrm{g} / \mathrm{L}$ and troponin I $24.6(\mathrm{NR}<0.04) \mu \mathrm{g} / \mathrm{L}$. Chest radiography was unremarkable (Fig. 4). In view of the unexplained hypoxia, computed tomography (CT) of the pulmonary arteries was done, which showed no pulmonary embolism. There was scarring with mild atelectasis in the lung bases, which was insignificant in causing his refractory hypoxia. On CT, there was reflux of contrast into the inferior vena cava and hepatic veins, in keeping with a right-heart failure. The clinical impression was a likely evolved inferior RV STEMI with cardiogenic shock. As the patient was hypoxic with high-flow oxygen and had a borderline low BP, despite the late presentation of myocardial infarction, a decision was made to transfer him to the cardiac catheterisation lab for $\mathrm{PCl}$.

Coronary angioplasty showed double vessel disease with $50 \%$ stenosis of proximal left anterior descending artery (pLAD) and $100 \%$ stenosis of proximal RCA (pRCA). Seeing as the patient was hypoxic and hypotensive, balloon angioplasty of the pRCA lesion was performed, with $<30 \%$ residual stenosis post-procedure. He subsequently underwent a transthoracic echocardiography (TTE), which showed a PFO with severe biventricular dysfunction with an ejection fraction of $15 \%$. Right-to-left intra-atrial shunting through the PFO was confirmed on saline contrast study (i.e. 'bubble study') (Fig. 5).

Right-heart catheterisation showed right and left atrial mean pressures of $25 \mathrm{mmHg}$ and $21 \mathrm{mmHg}$, respectively. The decision was made to close the PFO with a $9 \mathrm{~mm}$ Amplatzer occluder rather than a PFO device due to the wide gap. Final transoesophageal echocardiography (TOE) with agitated saline bubble contrast study, however, still showed the right-to-left shunting within the first three cardiac cycles, but the patient's oxygen saturation improved from $88 \%$ to $95 \%$ on room air. Repeat TOE three days later showed marked improvement of

${ }^{1}$ Emergency Medicine Department, National University Health System, ${ }^{2}$ Department of Surgery, Yong Loo Lin School of Medicine, National University of Singapore, Singapore Correspondence: Dr Chua Mui Teng, Senior Resident, NUHS Emergency Department, National University Hospital, Emergency Medicine Department, 5 Lower Kent Ridge Road, Singapore 119074. mui_teng_chua@nuhs.edu.sg 


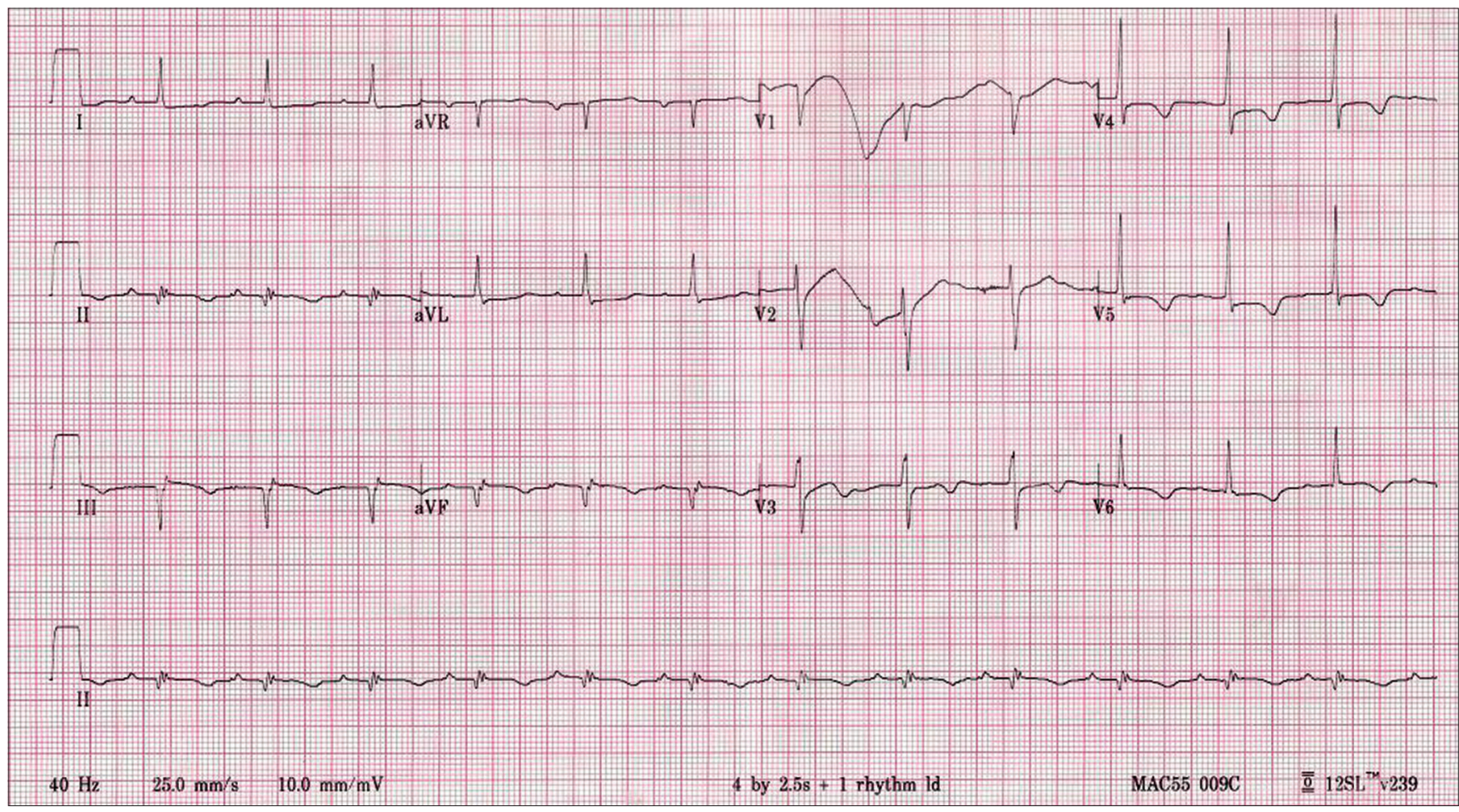

Fig. 1 ECG taken on the patient's arrival at the emergency department shows evolved inferior ST-elevation myocardial infarction.

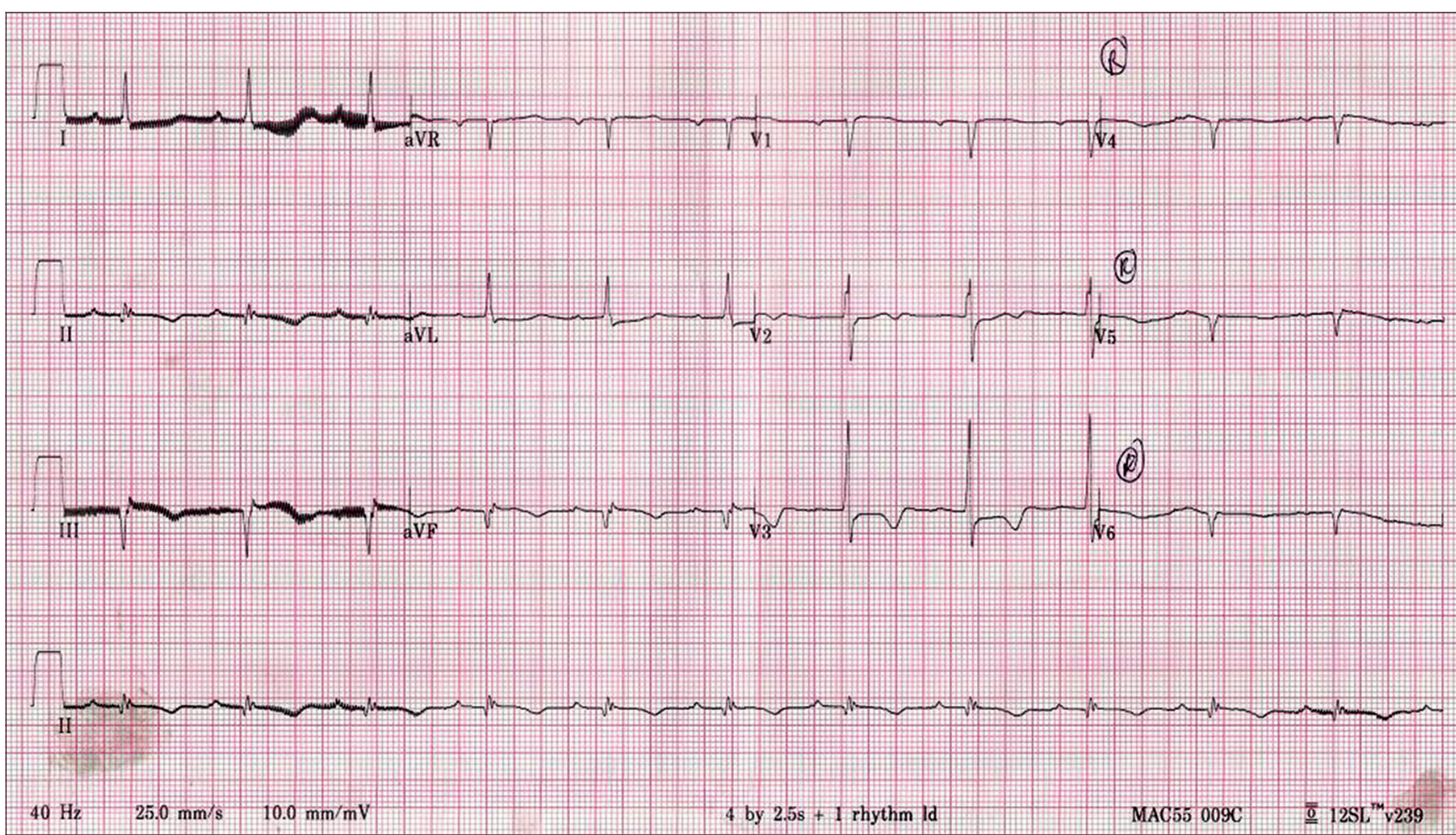

Fig. 2 Right-sided ECG taken on the patient's arrival at the emergency department shows evolved inferior ST-elevation myocardial infarction.

$R V$ function with no residual shunt. He was discharged well on Day 8 of admission.

\section{DISCUSSION}

Hypoxaemia is a recognised complication in acute myocardial infarction; it can occur due to pulmonary oedema from congestive cardiac failure in left ventricular infarct, pulmonary embolism or even due to an acute right-to-left shunting through a previously dormant PFO. ${ }^{(4)} \mathrm{A}$ dormant PFO has been described in up to $25 \%-30 \%$ of the general population, ${ }^{(5-7)}$ whereas fusion is complete at birth in the rest of the population. ${ }^{7}$

Patients with PFO remain asymptomatic, as the defect is a flap-like lesion and does not permit left-to-right cardiac 


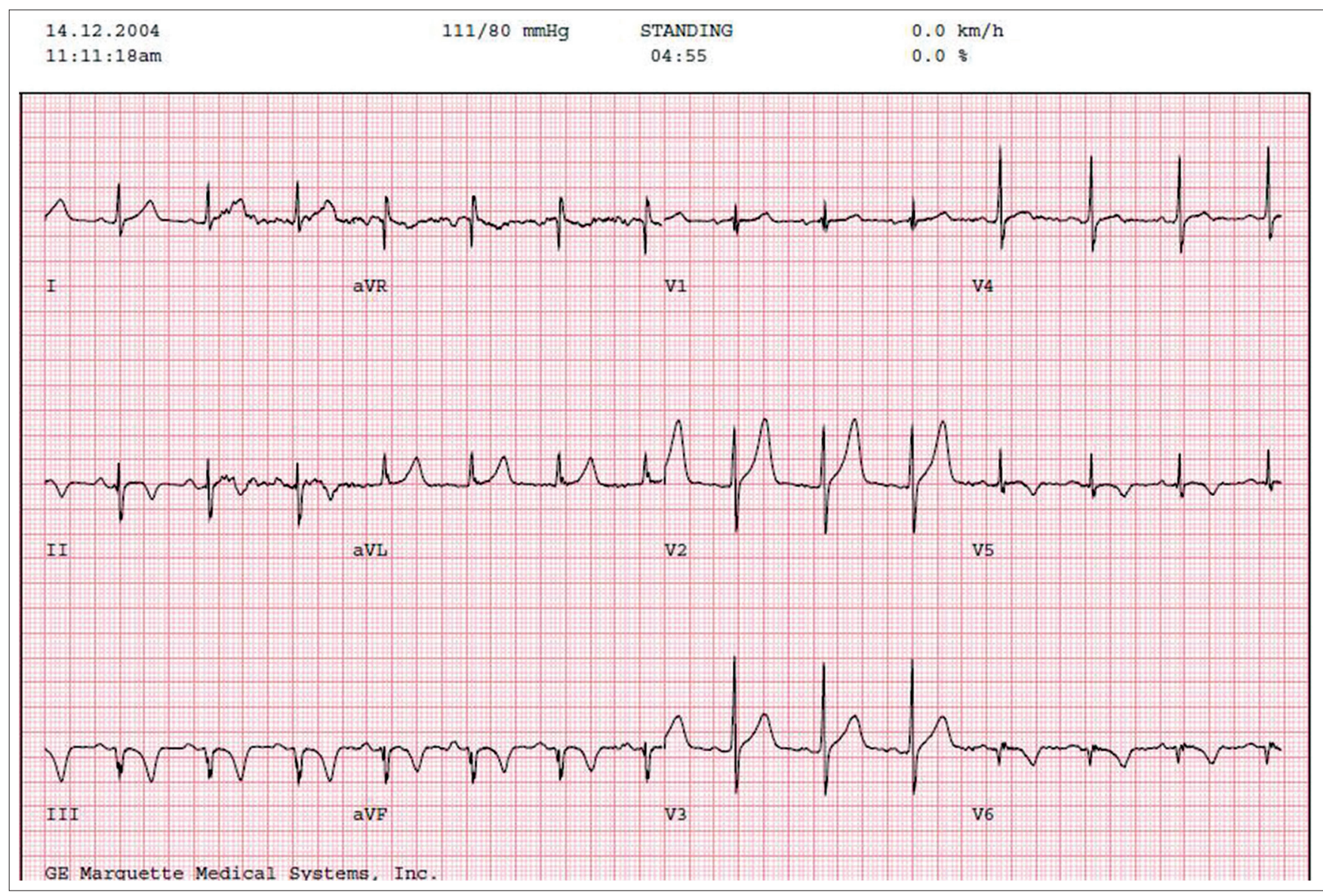

Fig. 3 Previous ECG taken in 2004.

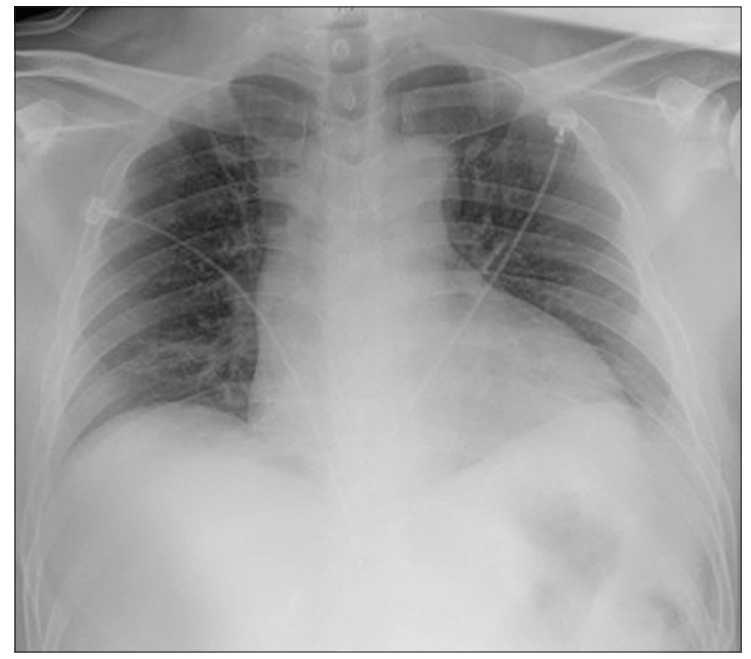

Fig. 4 Radiograph taken on the patient's arrival at the emergency department.

shunting normally ${ }^{(8)}$ when the atrial pressure is higher on the left than the right. During atrial contraction in ordinary circumstances, the right atrial pressure rises to $4-6 \mathrm{mmHg}$ whereas the left atrial pressure rises to about $7-8 \mathrm{mmHg} .{ }^{(9)}$ In RV infarction, the pressure in the right atrium increases to above that of the left atrium during inspiration when the right atrium is in diastole,,$^{(2,4)}$ and therefore results in a rightto-left inter-atrial shunting. The resultant effect is systemic hypoxaemia refractory to oxygen supplementation. This finding is consistent with that in our report - the patient's right atrial

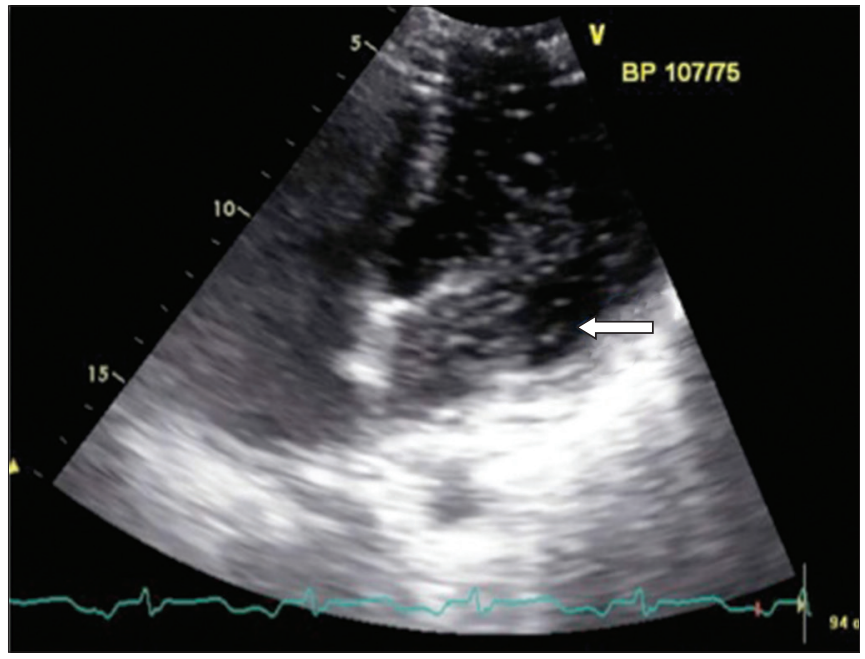

Fig. 5 Echocardiogram shows right-to-left shunted saline bubbles inside the left atrium (arrow).

mean pressure $(25 \mathrm{mmHg}$ ) exceeds the left atrial mean pressure (21 $\mathrm{mmHg}$ ).

Our patient has refractory hypoxia not corrected by high-flow oxygen in the absence of significant pulmonary pathology (clear chest radiography and no pulmonary embolism on CT pulmonary angiography [CTPA]). This should lead the attending physician to suspect the possibility of a shunt or haemoglobinopathy, such as methaemoglobinaemia, which is even rarer. The diagnosis of PFO resulting in right-to-left shunting could be clinched at bedside by performing echocardiography via a subcostal view 
Table I. A summary of dos and don'ts in an acute right-to-left shunt in right ventricular ST-elevation myocardial infarction.

\begin{tabular}{ll}
\hline Dos & Don'ts \\
\hline - Activate catheterisation & Provide positive ventilation, \\
lab for revascularisation & e.g. noninvasive ventilation or \\
- Start inotropes, e.g. & intubation \\
dobutamine & - Provide volume load \\
- Give pulmonary & - Administer nitrates \\
dilators, e.g. nitric oxide & - Use intra-aortic balloon pump \\
\hline
\end{tabular}

that demonstrates colour flow across the inter-atrial septum. The natural tendency to correct the hypotension would be to give small fluid boluses, but in the context of PFO with a right-to-left shunt, this could result in more shunting and worsening of the hypoxia. In such circumstances, it is best to start intravenous inotropes to improve the haemodynamics.

Hence, in RV infarction, it is essential to consider the presence of a right-to-left shunt when there is persistent uncorrectable hypoxaemia, especially if pulmonary embolism has been excluded with imaging. This consideration is crucial, as certain clinical managing principles need to be applied. Any measure that results in an increase in right-sided cardiac pressures and decreases afterload (which will cause a decrease in the left atrial pressure) will aggravate the right-to-left shunting. ${ }^{(4,10)}$ To avoid an increase in the right-sided cardiac pressure, the physician should avoid positive-end expiratory pressures ${ }^{(4,10)}$ and a volume load ${ }^{(10)}$ whenever possible. If the diagnosis is clear, inotropic agents or even cardiac pacing, if there is an atrioventricular block, may be more effective in cardiogenic shock. ${ }^{(10)}$ In patients with severe refractory hypoxia and cardiogenic shock, the physician may still need to institute positive pressure ventilation (via endotracheal intubation) and start inotropes before sending the patient to the catheterisation lab. Reducing afterload, and hence, left atrial pressure, which then increases shunt flow, is also contraindicated. Intra-aortic balloon pump insertion and nitrates should be avoided. ${ }^{(4,10)}$

Achieving revascularisation of the culprit lesion in acute myocardial ischaemia is the mainstay of treatment, ${ }^{(11)}$ as reperfusion improves the function of the right ventricle. Hence, the attending physician should recognise the need to activate the cardiac interventionist at the earliest possible time. Other measures to improve RV function, such as starting inotropic agent like dobutamine ${ }^{(11)}$ or using a pulmonary dilating agent like nitric oxide, may also be considered. ${ }^{(10,11)}$ Performing a CTPA in an acute STEMI with hypoxia within the first few hours of chest pain may not be appropriate, as it delays $\mathrm{PCl}$. However, in our patient, the symptoms were already two days old. Hence, we decided to proceed with CTPA to rule out pulmonary embolism as a cause of refractory hypoxia.

Therefore, in the setting of inferior myocardial infarction with $\mathrm{RV}$ involvement, it is important for the attending physician to consider the possibility of the presence of a PFO with right-to-left shunting if there is persistent hypoxaemia refractory to oxygen supplementation, so as to avoid medications or interventions that could worsen the shunt (Table I). Timely recognition of this condition can allow the physician in instituting definitive treatment by activation of cardiac interventionist to achieve coronary revascularisation, opting for medications that can improve RV function and preventing the worsening of the shunt by avoiding certain medications.

\section{REFERENCES}

1. Goldman L, Cook EF, Johnson PA, et al. Prediction of the need for intensive care in patients who come to the emergency departments with acute chest pain. N Engl J Med 1996; 334:1498-504.

2. Franco T, Melendez J, Malkin R, Schulman P. Acute right to left shunt through patent foramen ovale presenting as hypoxemia after myocardial infarction: a case report. Cases J 2009; 2:8878.

3. Kinch JW, Ryan TJ. Right ventricular infarction. N Engl J Med 1994; 330:1211-7.

4. Crawford LC, Panda M, Enjeti S. Refractory hypoxemia in right ventricular infarction: a case report. South Med J 2006; 99:79-81.

5. Kerut EK, Norfleet WT, Plotnick GD, Giles TD. Patent foramen ovale: a review of associated conditions and the impact of physiological size. J Am Coll Cardiol 2001; 38:613-23.

6. Chandrashekhar Y. PFO and the heart more than meets the eye! JACC Cardiovasc Imaging 2010; 3:840-2.

7. Hara H, Virmani R, Ladich E, et al. Patent foramen ovale: current pathology, pathophysiology, and clinical status. J Am Coll Cardiol 2005; 46: 1768-76.

8. Gomperts N, Fowler R, Horlick E, McLaughlin P. A broken heart: right-toleft shunt in the setting of normal cardiac pressures. Can J Cardiol 2008; 24:227-9.

9. Guyton AC, Hall JE. Textbook of Medical Physiology. 9th ed. Philadelphia: Saunders, 1996: 111.

10. Kuch B, Riehle M, von Scheidt W. Hypoxemia from right-to-left shunting through a patent foramen ovale in right ventricular infarction: treatment by revascularization, preload reduction, and, finally, interventional PFO closure. Clin Res Cardiol 2006; 95:680-4.

11. Hamid N, Keng F. Refractory hypoxia in right ventricular infarction. Ann Acad Med Singapore 2011; 40:325-6. 